\title{
A PINHOLE SUN SENSOR FOR BALLOON-BORNE EXPERIMENT ATTITUDE DETERMINATION
}

\author{
A. L. KOROTKOV*, M.-P. ENGLISH*, G. S. TUCKER ${ }^{*,}$, \\ E. PASCALE ${ }^{\dagger}$ and N. GANDILO $\ddagger$ \\ * Department of Physics, Brown University \\ 182 Hope Street, Providence, RI 02912, USA \\ ${ }^{\dagger}$ School of Physics and Astronomy, Cardiff University \\ 5 The Parade, Cardiff CF24 3AA, UK \\ ${ }^{\ddagger}$ Department of Astronomy and Astrophysics, University of Toronto \\ 50 St. George Str., Toronto, ON M5S 3H4, Canada \\ §gregory_tucker@brown.edu
}

Received 2013 May 30; Revised 2013 June 20; Accepted 2013 July 14; Published 2013 August 6

\begin{abstract}
We report on the design, calibration and in-flight performance of a sun sensor, which is used to determine the attitude of a balloon-borne telescope. The device uses a position-sensitive detector (PSD) in a pinhole camera. By determining the position of the image of the Sun on the PSD, the orientation of the sun sensor and the boresight of the telescope relative to the Sun can be determined. The pinhole sun sensor (PSS) was first flown in the December 2010 flight of the Balloon-borne Large Aperture Submillimeter Telescope with Polarization (BLAST-Pol). In flight the PSS achieved an accuracy (combined azimuth and elevation) of about $0.18^{\circ}$. The accuracy could be improved by increasing the distance between the pinhole and the PSD, but the field-of-view of the PSS would be reduced.
\end{abstract}

Keywords: Balloons, instrumentation: miscellaneous, techniques: miscellaneous.

\section{Introduction}

A sun sensor is often used in the attitude control systems of balloon-borne experiments (Pascale et al., 2008; Tamura et al., 2003; Quine et al., 2002; Romeo et al., 2001). During circumpolar flights, when the Sun is always above the horizon, a sun sensor provides reliable and accurate attitude determination.

Many experiments use magnetometers to determine azimuth, but when flying near a magnetic pole of the Earth, the accuracy of a magnetometer is limited even when corrected by the best magnetic field models since solar activity can influence the magnetic field. The Global Positioning System (GPS) can also be used to determine azimuth. Some experiments rely on daytime star cameras and gyroscopes to provide highly accurate attitude determination, but not all fields of the sky have sufficient quantities of bright stars to provide pointing solutions, and in every flight of the BLAST-Pol experiment at least one star camera has failed. A sun sensor thus provides an important complement to the pointing sensors commonly used in balloonborne experiments.

The PSS provides its orientation relative to the Sun. If the position of the Sun in the horizontal coordinate frame is known, which requires knowing the latitude and longitude of the experiment as well as the date and time, then it is possible to determine the azimuth of the gondola. Ideally the gondola remains level during flight, but a PSS can also provide a determination of the pitch or roll angle of the gondola, depending on the orientation of the PSS.

We describe the design and performance of a sun sensor which uses a position sensitive detector (PSD) in a pinhole camera. By measuring the position of the image of the Sun on the PSD, the angular position of the Sun relative to the sun sensor can be determined. 


\section{A. L. Korotkov et al.}

\section{Design}

Sun sensor designs for attitude determination have used a number of approaches, which we briefly describe here. In one approach a sun sensor is made using a vertical and a horizontal slit. Behind each slit is mounted a linear sensor (linear CCD (Pascale et al., 2008; Romeo et al., 2001) or solar cell (Quine et al., 2002)) in a direction orthogonal to the corresponding slit. Another approach has been to use a total power sensor such as a CdS cell (Romeo et al., 2001) or photodiode (Pascale et al., 2008), where the response of the detector depends on the cosine of the incident angle of the sun. The use of a four-quadrant photodiode to electromechanically track the sun has also been considered (Romeo et al., 2001). Tamura et al. (2003) developed a sun sensor using a pinhole and PSD similar to the one we describe here, but which only had 1 degree resolution.

The sensitive element in the PSS is a Hamamatsu S5991-01 two-dimensional PSD. The PSD is a PIN silicon structure with $9 \mathrm{~mm} \times 9 \mathrm{~mm}$ active area and spectral sensitivity from $320 \mathrm{~nm}$ to $1100 \mathrm{~nm}$. The peak sensitivity is at $960 \mathrm{~nm}$. A reverse bias $V_{R}$ is applied between the top highly p-doped layer and bottom n-doped layer. In the dark, there are practically no carriers in the intrinsic layer, which results in a dark current less than $50 \mathrm{nA}$. The absorbed light generates charge carriers (electron and holes) in the intrinsic layer and produces a photocurrent. The resistance of the bottom layer determines the distribution of the current between four anodes placed in the corners of the active area. In the linear regime, this distribution depends on the position of the light spot, its size and shape, and on the configuration of the contact pads. The manufacturer provides an approximation for the position of the light spot on the PSD as a function of the measured currents $I_{1}, I_{2}, I_{3}$ and $I_{4}$ :

$$
\begin{aligned}
& x=\frac{L}{2} \frac{\left(I_{2}+I_{3}\right)-\left(I_{1}+I_{4}\right)}{\left(I_{1}+I_{2}+I_{3}+I_{4}\right)}, \\
& y=\frac{L}{2} \frac{\left(I_{2}+I_{4}\right)-\left(I_{1}+I_{3}\right)}{\left(I_{1}+I_{2}+I_{3}+I_{4}\right)},
\end{aligned}
$$

where $L=10 \mathrm{~mm}$. The manufacturer specifies the typical position detection error for a circular light spot of diameter $200 \mu \mathrm{m}$ to be $150 \mu \mathrm{m}$ with a position resolution of $1.5 \mu \mathrm{m}$. We find the position detection error to be significantly smaller than $150 \mu \mathrm{m}$ when the light spot is within $80 \%$ of the center-to-edge distance of the active area and to

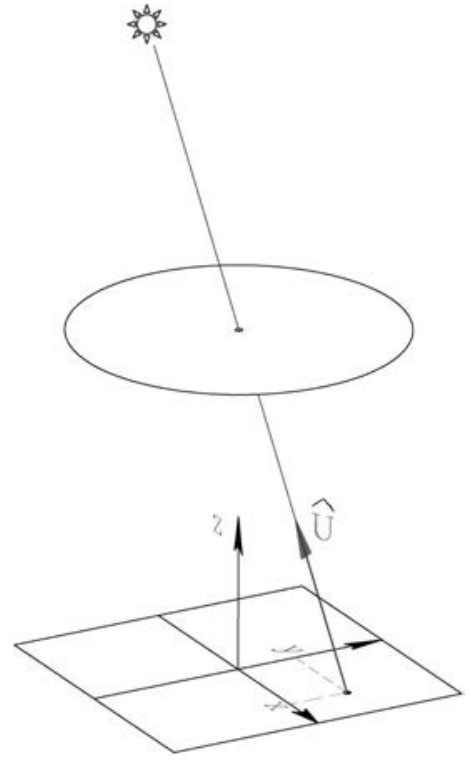

Fig. 1. The geometry of the PSS. The PSD is located behind a pinhole. The Sun illuminates a spot at position $(x, y)$ on the PSD. $\hat{U}$ is the unit vector pointing to the Sun. In the PSD Cartesian frame, $\hat{U}$ is determined by Eq. (2). In practice we try to mount the PSS to the gondola so that the $x$-axis of the PSD is horizontal and the roll angle $\psi$ is small (see text).

increase as the light spot approaches an edge or corner of the PSD.

In practice we apply a reverse bias of $V_{R}=0 \mathrm{~V}$. The four currents are converted to voltages using four OP-27GP operational amplifiers in a transimpedance configuration. Although the PSD itself is fast, with a rise time of $2 \mu \mathrm{s}$, the BLAST-Pol flight computer reads and records the four resulting voltages at $5 \mathrm{~Hz}$.

Figure 1 shows the geometry of the PSS, and Fig. 2 shows a photograph of a PSS. The PSD was placed in a blackened aluminum chamber $D=10 \mathrm{~mm}^{(\mathrm{a})}$ behind a $200 \mu \mathrm{m}$ diameter pinhole (Edmund Optics NT39-894). This pinhole was chosen since one side is black. This configuration provides a FOV of about $45^{\circ}$ in both the "horizontal" ( $x$-axis of the PSD) and "vertical" ( $y$-axis of the PSD) directions; the FOV is set by the size of the PSD and the distance between the pinhole and the PSD. When the Sun is in the FOV of the PSS, the position of the light spot on the PSD is calculated using Eq. (1). The direction to the Sun can then be calculated. If the $z$-axis of the PSD is directed towards the pinhole, then the unit vector

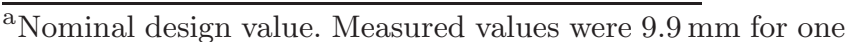
PSS (PSS1) and $10.3 \mathrm{~mm}$ for the other PSS (PSS2).
} 


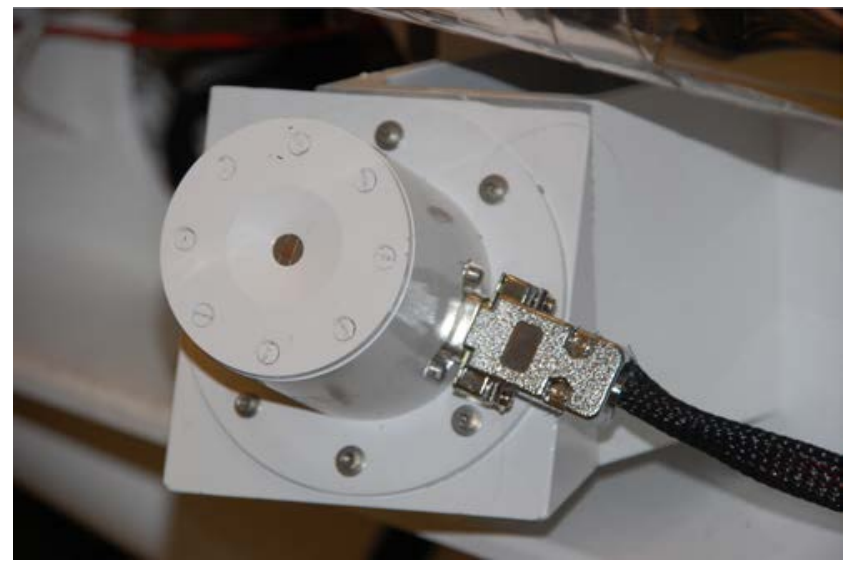

Fig. 2. A photograph of a PSS attached to the outer frame of the BLAST-Pol experiment. The PSS is painted white to help prevent overheating. The transimpedance amplifiers are also located in the PSS case.

in the direction of the Sun is given by

$$
\hat{U}=\left(\begin{array}{c}
-\left(x-x_{0}\right) / k \\
-\left(y-y_{0}\right) / k \\
D / k
\end{array}\right),
$$

where $k=\sqrt{\left(x-x_{0}\right)^{2}+\left(y-y_{0}\right)^{2}+D^{2}}$, and $x_{0}$ and $y_{0}$ are the lateral offsets of the pinhole relative to the center of the PSD.

The position of the light spot and corresponding vector $\hat{U}$ is determined from the four voltages using Eqs. (1) and (2). In the 2010 flight of BLAST-Pol one PSS (PSS1) had an azimuth angle $\phi=60^{\circ}$ relative to the boresight of the telescope, and the other PSS (PSS2) had an azimuth angle of $\phi=135^{\circ}$. Both PSSs were tilted up in elevation by an angle $\alpha=25^{\circ}$ to ensure that the Sun was visible and to avoid reflections from the Antarctic ice. We found it was important to position the PSSs on the gondola in such a way as to avoid having gondola elements in the PSS FOV.

\section{Calibration and Accuracy}

The accuracy of the PSS is determined by several factors including the size and shape of the light spot on the PSD and a parasitic contribution from scattered light in the atmosphere onto the PSD. Increasing the distance between the pinhole and PSD reduces the FOV but increases the resulting angular accuracy.

Pre-flight calibration was carried out on the ground using the Sun. A PSS was attached to a rotary stage which repeatedly scanned the PSS in elevation by $90^{\circ}$ at a rate of $1.5^{\circ} / \mathrm{s}$ while the azimuth angle was fixed. Azimuthal coverage was provided as the Sun moved across the sky. The PSS signal was sampled at $10 \mathrm{~Hz}$. The expected sun position relative to the PSD was calculated for each sample:

$$
\hat{U}_{e}=R_{z}(\psi) R_{x}(\alpha) R_{y}\left(\phi_{0}-\phi_{S}\right) R_{x}\left(-\alpha_{S}\right) \hat{U}_{0},
$$

where $\hat{U}_{0}=(0,0,1)$ is a unit vector along the PSS optical axis, $R_{x}, R_{y}, R_{z}$ are the elemental rotation matrices in a Cartesian frame, $\phi_{S}$ and $\alpha_{S}$ are the Sun azimuth and elevation, $\phi_{0}$ is the fixed PSS azimuth, $\alpha$ is the elevation angle, and $\psi$ is the roll angle of the PSS about its optical axis. The elemental rotation matrices are defined as follows:

$$
\begin{aligned}
& R_{x}(\zeta)=\left(\begin{array}{ccc}
1 & 0 & 0 \\
0 & \cos \zeta & -\sin \zeta \\
0 & \sin \zeta & \cos \zeta
\end{array}\right), \\
& R_{y}(\zeta)=\left(\begin{array}{ccc}
\cos \zeta & 0 & \sin \zeta \\
0 & 1 & 0 \\
-\sin \zeta & 0 & \cos \zeta
\end{array}\right), \\
& R_{z}(\zeta)=\left(\begin{array}{ccc}
\cos \zeta & -\sin \zeta & 0 \\
\sin \zeta & \cos \zeta & 0 \\
0 & 0 & 1
\end{array}\right) .
\end{aligned}
$$

During calibration $\alpha$ is read by the rotary stage encoder, and during flight $\alpha$ is fixed. In practice we try to align the PSD $x$-axis horizontally to keep the roll angle small $\left(|\psi| \lesssim 1^{\circ}\right)$, but this is not strictly necessary.

The scanning procedure allowed practically the entire active area of the PSD to be covered, minimizing possible bias in the mean error. The expected direction of the Sun and corresponding position of the light spot on the active area of the PSD were compared with the calculated $x$ and $y$ coordinates of a light spot on the PSD derived from Eqs. (1) and (2). The discrepancy between the expected and measured coordinates of the light spot, or linear error, was calculated as a function of the light spot position. This error can also be expressed in angular terms as a difference between actual pointing and that derived from the light spot position. The uncertainties in mechanical factors: the pin-hole coordinates in the PSD frame (the distance from the PSD surface to the pinhole and the lateral offsets), roll angle $\psi$ between the PSD $x$-axis and the horizon, as well as azimuth and elevation offsets were treated as fit parameters within their corresponding tolerance ranges, and chosen to null 


\section{A. L. Korotkov et al.}

the mean and minimize the rms value of the discrepancy for the entire observation.

When the light spot is within $0.5 \mathrm{~mm}$ of the edge, part of the light spot falls outside the sensitive area, reducing the total response current and shifting the "measured" position towards the PSD center. This area was excluded from the analysis. An upper limit of $200 \mu \mathrm{m}$ on the pin-hole diameter was set to keep this dead area small, but this also limits the total photocurrent produced in the PSD to $5 \%$ of PSD saturation level. In practice, the position determination is performed when the total response current exceeds a certain valid level and the light spot position is within $4 \mathrm{~mm}$ from the center of the PSD in both the $x$ - and $y$-directions.

The atmosphere is considerably brighter on the ground than at float $(\sim 40 \mathrm{~km})$ during the balloon flight. On the ground the brightness of the atmosphere depends strongly on the precipitable water vapor, so calibration is done on nearly cloudless days with low humidity. On the ground the total sky brightness within the PSS FOV and in the spectral range of the PSD is estimated using MODTRAN to be $3-5 \%$ of the Sun brightness, depending on atmospheric conditions and the elevation of the Sun. In our model, we use the brightness of Sun on the ground and integrate the sky brightness results of the MODTRAN model in the field of view of the PSS. The net effect of sky brightness, since it adds to all of the currents in Eq. (1), is to make the measured Sun position closer to the center of the PSD than would be expected with no sky brightness. We treat this by using a ground-derived pinhole-to-PSD distance $D_{\text {ground }}$. The contribution from the atmospheric brightness at $40 \mathrm{~km}$ to the PSS signal is negligible, and we use the measured pinhole-to-PSD distance $D$. Removing the estimated sky brightness contribution from the ground-based calibration provides an initial estimate for the expected response of the PSS at an altitude of $40 \mathrm{~km}$.

Figure 3(a) shows the total photo-current in PSS2 as a function of the measured light spot position on the active surface. The vertical stripes of lower intensity correspond to times when light clouds or haze reduced the intensity of sun on the PSD. The expected position of the light spot was calculated, based on the rotary stage elevation angle and the sun position on the sky. Figure 3(b) show the angular error obtained from the calibration procedure as a function of position on the PSD. Figures 3(c) and 3(d) show the linear errors for $x$ - and $y$-directions, respectively.
The errors increase when the total current drops due to the clouds.

For a total PSD current exceeding $9.3 \mu \mathrm{A}$, the rms azimuth and elevation angle error are $0.14^{\circ}$ and $0.17^{\circ}$, respectively averaged across the active area of the PSD. The total angular error (combined for the two directions) is $0.22^{\circ}$. This error is about three times smaller than the expected error from the manufacturer-specified PSD position error of $150 \mu \mathrm{m}$, probably due to the compensating effect of the light spot shape. It is higher though than the error that could be associated with the PSD resolution $(1.5 \mu \mathrm{m})$ due to the significant effect of sky brightness.

The best fit value for the distance between the pinhole and the PSD appeared to be $3 \%$ less than the actual one due to the contribution to the photo-current from the light scattered by the atmosphere. This additional photo-current, being distributed among the four contacts, shifts the measured light spot position towards the center of the PSD as though the actual spot were shifted if the pinhole were closer to the PSD. Obtaining a more accurate calculation of the effect from a groundbased calibration test would be difficult and would require knowing the exact shape of the PSD contacts; this information is not provided on the manufacturer specification sheet.

The PSD dark current and transimpedance circuits results in an estimated angular error of a few arcminutes.

\section{In-Flight Performance}

In the 2010 flight of the BLAST-Pol experiment (Fissel et al., 2010) from the Antarctic two pinhole sun sensors (PSSs) were attached rigidly to the outer frame of the payload (or gondola) and were used to determine the azimuth of the instrument. The inner frame of the gondola, to which the telescope is attached, slews in elevation. Each PSS has a field-of-view (FOV) of $45^{\circ}$, which is determined by the size of the PSD and the distance between the PSD and the pinhole. The orientation of each sun sensor was chosen so that the sun would be in the FOV of the PSS when the BLAST-Pol telescope was observing a target source. In the 2012 flight of BLAST-Pol, we used a total of four PSSs to provide approximately $180^{\circ}$ of azimuth coverage. In this paper, we present only data from the 2010 flight, but the sun sensors appear to have performed comparably during the 2012 flight. Since both of 


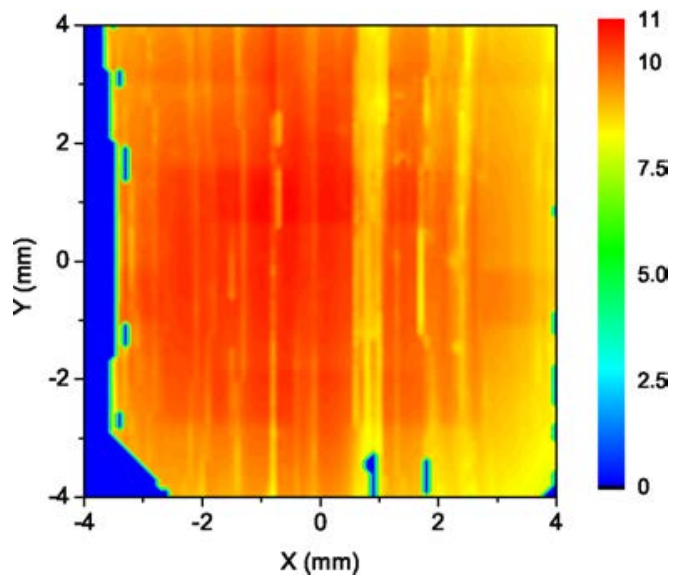

(a)

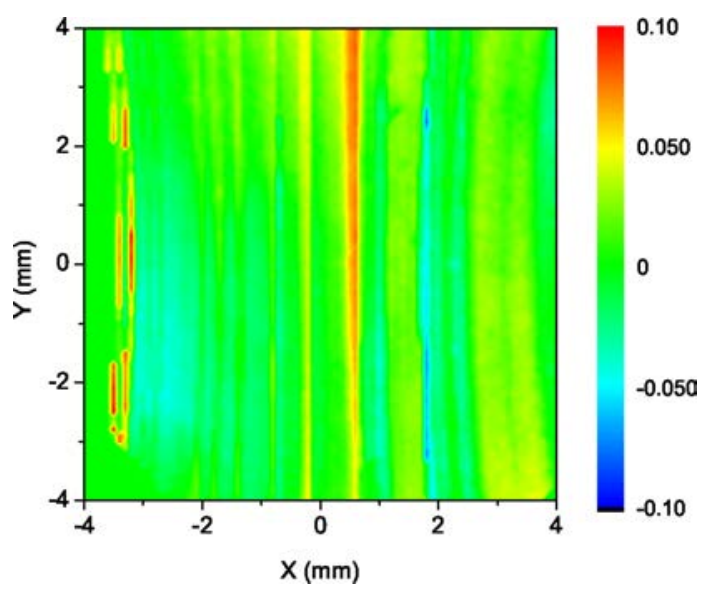

(c)

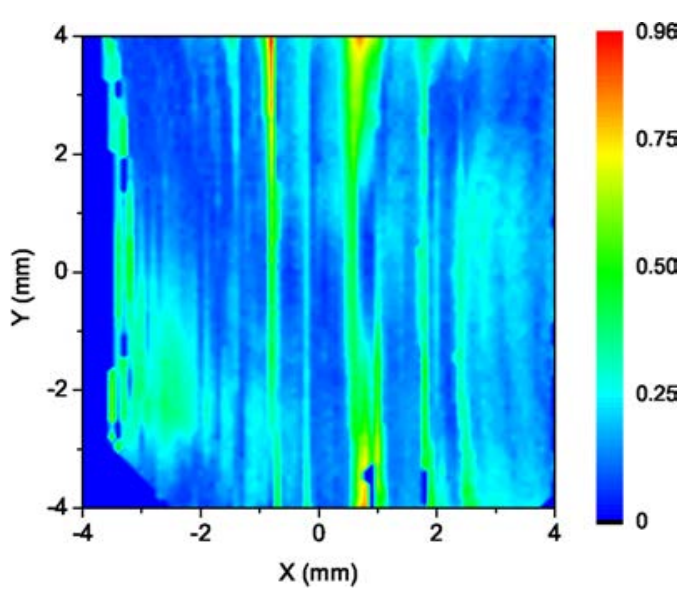

(b)

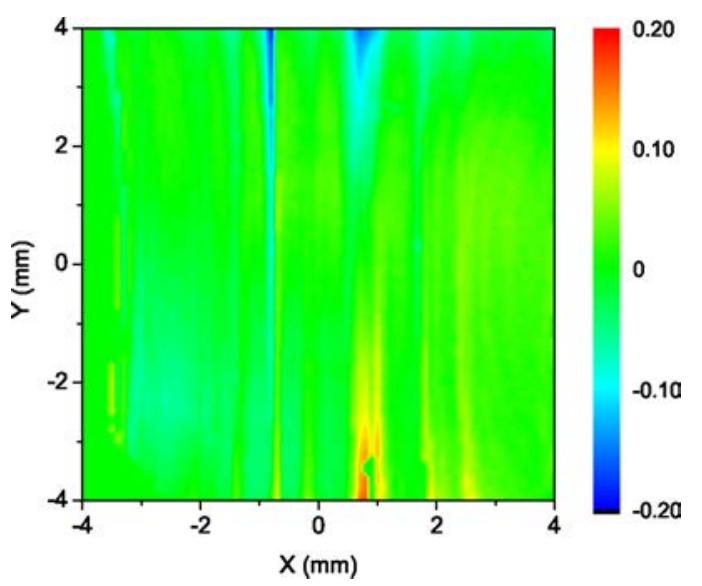

(d)

Fig. 3. Results obtained from calibration of the PSS. (a) The upper left panel shows the total photo-current from the PSD as a function of the Sun light spot position on the PSD. The vertical stripes of lower intensity correspond to light clouds or haze occasionally reducing the direct sun light intensity. Scale is in $\mu \mathrm{A}$. (b) The upper right panel shows the resulting angular error obtained from the calibration procedure. Scale is in degrees. (c) The lower left panel indicates the linear error in the $x$-direction while $(\mathrm{d})$ the lower right panel indicates the linear error in the $y$-direction. In both panels the scale is in degrees.

the redundant star cameras failed during the 2012 flight, the sun sensors became critical for postflight pointing reconstruction in addition to in-flight pointing. During flight, the light spot position on the PSD is determined by the instrument scan strategy; the spot traces did not cover the active area uniformly.

The gondola azimuth, Az, was calculated from the PSS azimuth relative to the Sun, $\phi_{\mathrm{RS}}$, and the known Sun azimuth, $\phi_{\mathrm{S}}$ :

$$
\mathrm{Az}=\phi_{\mathrm{S}}+\phi_{\mathrm{RS}}-\phi,
$$

where

$$
\begin{gathered}
\phi_{\mathrm{RS}}=\arctan \left(\frac{\hat{W}_{x}}{\hat{W}_{z}}\right), \\
\hat{W}=R_{x}(-\alpha) R_{z}(-\psi) \hat{U} .
\end{gathered}
$$

The elevation angle of the Sun can also be calculated:

$$
\mathrm{El}_{\mathrm{S}}=\arctan \left(\frac{\hat{W}_{y}}{\sqrt{\hat{W}_{x}^{2}+\hat{W}_{z}^{2}}}\right) .
$$

Comparison with the actual sun elevation could also be used to determine the gondola pitch or roll depending on the orientation of the PSS on the gondola.

The rms angular error in azimuth was $0.10^{\circ}$ and $0.13^{\circ}$ and in elevation $0.11^{\circ}$ and $0.08^{\circ}$, for PSS1 and PSS2, respectively. Lateral pin-hole linear offsets, elevation and azimuth angular offsets, and roll angle were considered as fit parameters. Distance from the PSD to the pinhole was assumed equal to the measured value and was not considered as a fit 


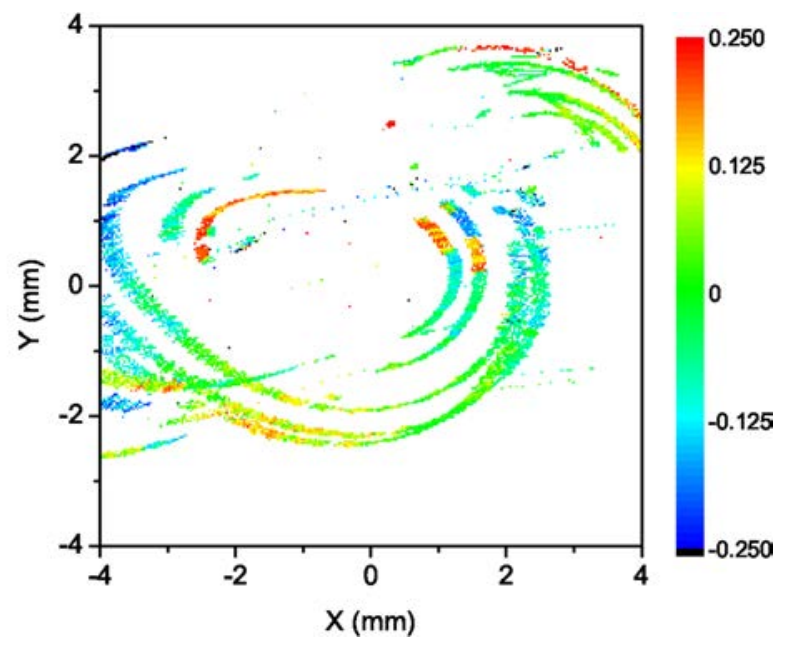

(a)

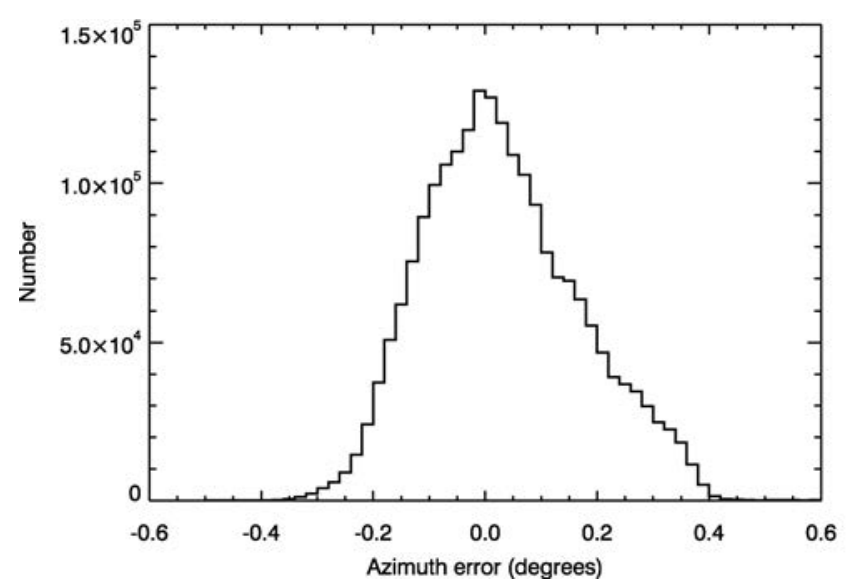

(c)

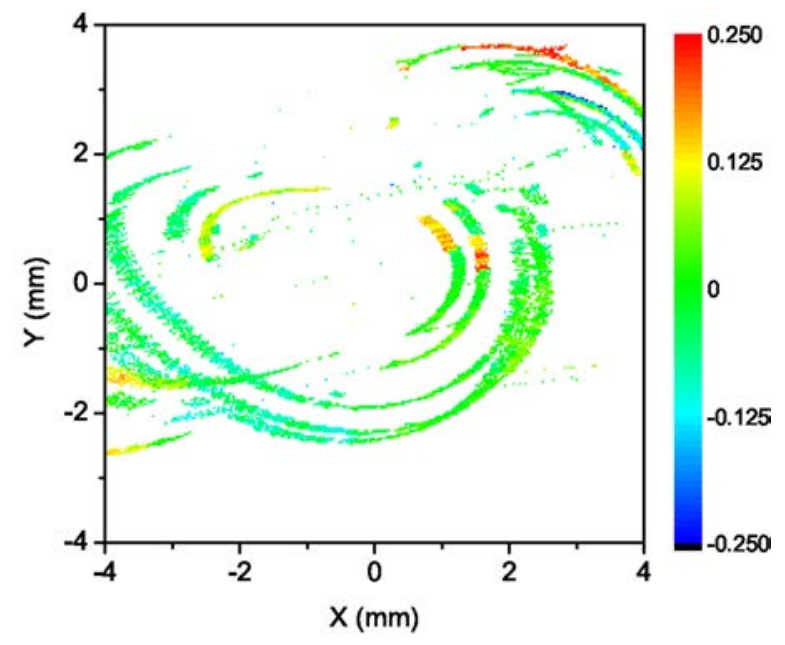

(b)

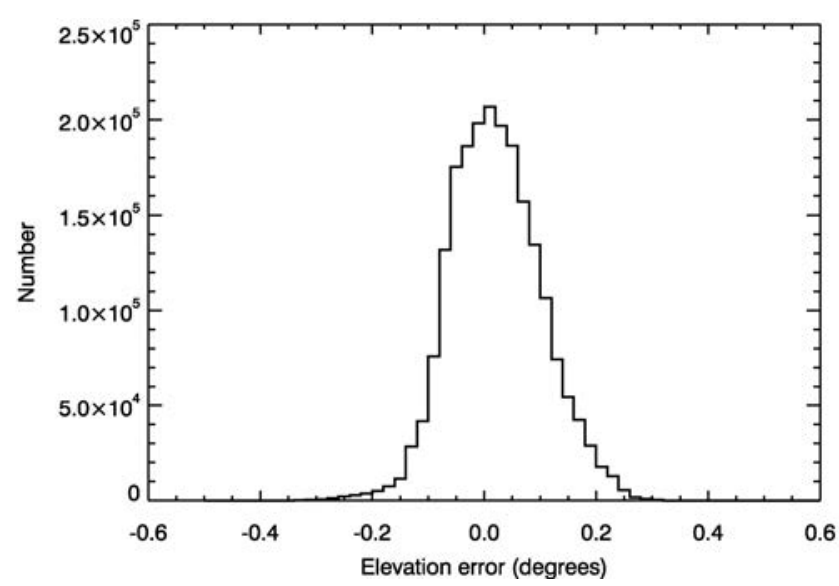

(d)

Fig. 4. Azimuth and elevation errors of a PSS obtained during the 2010 Antarctic flight. (a) The upper left panel shows the azimuth error of the PSS as determined by post-flight attitude reconstruction which uses star cameras. (b) The upper right panel shows the corresponding errors in elevation determined in the same way. (c) The lower left panel shows the histogram of the azimuth errors of the upper left panel, and (d) the lower right panel shows the corresponding elevation error histogram.

parameter. Figure 4 shows the azimuth and elevation error histograms for PSS2. Since we were not concerned with the pitch or roll of the gondola, which can have amplitudes of $\sim 1^{\circ}$, the azimuth and elevation errors are larger in flight than those during the ground calibration due to the gondola pendulations.

During the BLAST-Pol 2010 Antarctic flight, at altitudes of about $40 \mathrm{~km}$, the PSSs determined angular positions (combined azimuth and elevation) accurately to $0.18^{\circ}$. The influence of the skyscattered light at such altitudes is two orders smaller than on the ground. Post-flight analysis of the PSS performance was done by comparing with the post-flight attitude reconstruction, which relies on information from star cameras and gyros.

\section{Conclusions}

A pinhole sun sensor was designed and successfully tested during the 2010 Antarctic flight of BLAST-Pol. The PSS is a compact device and is practically insensitive to spurious reflected light originating outside of its well defined field of view. The demonstrated accuracy is better than $0.18^{\circ}$ in both horizontal and vertical directions. The accuracy is determined mostly by the PSD position error, shape of the light spot, and the scattered 
light from sky. Ground-based calibration to the accuracy of the PSD resolution is problematic due to the relatively high sky brightness. The BLASTPol PSS FOV was $45^{\circ}$, but this could be increased or decreased by changing the distance between the PSD and the pinhole, with a corresponding decrease or increase in accuracy. In the 2012 flight of BLAST-Pol we used two additional PSSs to provide azimuthal coverage of about $180^{\circ}$; the performance of the sun sensors during this flight appears to be consistent with that in the 2010 flight.

\section{Acknowledgments}

We acknowledge the support of NASA, the NASA Rhode Island Spacegrant, the NSF Office of Polar
Programs, the Canadian Space Agency, the Natural Sciences and Engineering Research Council (NSERC) of Canada, and the UK Science and Technology Facilities Council (STFC).

\section{References}

Fissel, L. M. et al., 2010, Millimeter, Submillimeter, and FarInfrared Detectors and Instrumentation for Astronomy $V$., in Proc SPIE 7741, eds. Holland, W. S. \& Zmuidzinas, J., 77410E-77410E-14.

Pascale, E. et al., 2008, Ap. J., 681, 415.

Quine, B. M. et al., 2002, J. Atmospheric and Oceanic Technology, 19, 618 .

Romeo, G. et al., 2001, AIP Conf. Proc., 616, 59.

Tamura, T. et al., 2003, Yukio Report of the National Astronomical Observatory of Japan, 6, 117. 